\title{
Partial Redistribution Effects on Polarized Lines Formed in Moving Media in the Presence of a Weak Magnetic Field
}

\author{
M. Sampoorna and K. N. Nagendra \\ Indian Institute of Astrophysics, Koramangala, Bengaluru 560 034, India \\ email: sampoorna@iiap.res.in, knn@iiap.res.in
}

\begin{abstract}
Macroscopic velocity fields in stellar atmospheres significantly affect the shapes of the emergent Stokes profiles. The inextricable coupling between the angle and frequency variables becomes more complex in a moving medium when compared to a static medium. In this paper we consider both complete frequency redistribution (CRD) and partial frequency redistribution (PRD) in the line scattering of a two-level atom in the presence of an external weak magnetic field. For simplicity we consider empirical velocity laws to represent motion of the atmospheric layers. We present emergent Stokes profiles computed with CRD, angle-averaged $\mathrm{PRD}$, and angle-dependent PRD. We show that angle-dependent PRD effects are important both in non-magnetic and magnetized scattering when vertical velocity gradients are present in the atmosphere. The results are presented for simple atmospheric models. They are expected to be of relevance to polarized line formation in slowly expanding chromospheric layers.
\end{abstract}

Keywords. Polarization, radiative transfer, scattering, stars: atmospheres, Sun: chromosphere

\section{Introduction}

Mass motions are common in astrophysical objects such as stellar winds, novae, supernovae, etc. Such macroscopic velocity fields in spectral line formation regions strongly affect the shape of the spectral line. For example an expanding atmosphere produces so-called P-Cygni profiles with a blue absorption and a red emission, instead of an absorption line. While detailed studies on the effect of macroscopic velocity fields on the unpolarized radiation field exists, little has been explored with regard to their effect on the polarized radiation field, especially when the effects of partial frequency redistribution (PRD) and a weak magnetic field are taken into account.

Early works on non-magnetic resonance line polarization in moving media were carried out by Sengupta (1993) and Nagendra (1996), who assumed complete frequency redistribution (CRD) and PRD in line scattering, respectively. Recently, Carlin et al. (2012, 2013) presented a detailed study on the effect of macroscopic vertical velocity fields on the scattering polarization profiles of the Ca II IR triplet lines, in isothermal and realistic solar model atmospheres, and also in hydrodynamical models of the Sun. To this end they self-consistently solved the polarized statistical equilibrium equation and the transfer equation for a five level Ca II model atom under the approximation of CRD. They show that the vertical velocity gradients significantly affect the polarization profiles of the Ca II IR triplet lines by producing Doppler shifted asymmetric line profiles and by considerably modifying the polarization amplitudes compared to the static or constant velocity case.

In the present paper we study the effect of macroscopic vertical velocity fields on the linear polarization profiles formed due to resonance scattering in an isothermal atmosphere. We take into account the effects of PRD as well as a weak magnetic field (Hanle 
effect). We consider a two-level atom model with an infinitely sharp and unpolarized lower level. In Section 2 we recall the polarized PRD transfer equation in the observer's frame. The numerical method for solving it is briefly outlined in Section 3. Results of our numerical study are presented in Section 4. Conclusions are presented in Section 5.

\section{Polarized PRD Transfer Equation in the Observer's Frame}

We consider a one-dimensional planar isothermal atmosphere with velocity fields along the atmospheric normal. The polarized transfer equation in the observer's frame is

$$
\mu \frac{\partial \boldsymbol{I}(\tau, x, \boldsymbol{\Omega})}{\partial \tau}=[\varphi(x, \mu)+r][\boldsymbol{I}(\tau, x, \boldsymbol{\Omega})-\boldsymbol{S}(\tau, x, \boldsymbol{\Omega})],
$$

where $\boldsymbol{I}=(I, Q, U)^{\mathrm{T}}$ is the Stokes vector, $\boldsymbol{\Omega}(\theta, \chi)$ is the ray direction with respect to the atmospheric normal, $\mu=\cos \theta$, and $\tau$ is the line optical depth. The normalized Voigt function is $\varphi(x, \mu)=H(a, x-\mu V)$, where $x=\left(\nu-\nu_{0}\right) / \Delta \nu_{\mathrm{D}}\left(\nu_{0}\right.$ being the line center frequency and $\Delta \nu_{\mathrm{D}}$ the Doppler width) and $V$ is a non-dimensional velocity defined as $V=\left(\nu_{0} / c\right) v_{z} / \Delta \nu_{\mathrm{D}}\left(v_{z}\right.$ being the vertical velocity field $)$. The ratio of continuum to line absorption coefficient is denoted by $r$. The reference direction for positive Stokes $Q$ is chosen to be perpendicular to the nearest limb. The total source vector is given by

$$
\boldsymbol{S}(\tau, x, \boldsymbol{\Omega})=\frac{\varphi(x, \mu) \boldsymbol{S}_{l}(\tau, x, \boldsymbol{\Omega})+r \boldsymbol{S}_{c}}{\varphi(x, \mu)+r},
$$

where the unpolarized continuum source vector is given by $\boldsymbol{S}_{c}=B_{\nu_{0}} \boldsymbol{U}$, with $B_{\nu_{0}}$ the Planck function at the line center and $\boldsymbol{U}=(1,0,0)^{\mathrm{T}}$. The line source vector has the form

$$
\boldsymbol{S}_{l}(\tau, x, \boldsymbol{\Omega})=\epsilon B_{\nu_{0}} \boldsymbol{U}+\int_{-\infty}^{+\infty} \frac{1}{4 \pi} \oint \frac{\mathbf{R}\left(x, \boldsymbol{\Omega}, x^{\prime}, \boldsymbol{\Omega}^{\prime}, \boldsymbol{B}\right)}{\varphi(x, \mu)} \boldsymbol{I}\left(\tau, x^{\prime}, \boldsymbol{\Omega}^{\prime}\right) \sin \theta^{\prime} d \theta^{\prime} d \chi^{\prime} d x^{\prime}
$$

where $\boldsymbol{\Omega}^{\prime}\left(\theta^{\prime}, \chi^{\prime}\right)$ is the incident ray direction with respect to the atmospheric normal, and $\epsilon$ is the thermalization parameter. In the presence of a weak vector magnetic field, $\boldsymbol{B}$, the exact Hanle redistribution matrix, $\mathbf{R}$, is given by Approximation-I of Bommier (1997). The explicit expression of both the angle-dependent (AD) and angle-averaged (AA) versions of this matrix can be found in Nagendra \& Sampoorna (2011) (see also Sampoorna 2014) and Sampoorna et al. (2008), respectively. In the limit of CRD, the matrix $\mathbf{R}$ is given by $\mathbf{R}=\varphi(x, \mu) \varphi\left(x^{\prime}, \mu^{\prime}\right) \mathbf{P}_{\mathrm{H}}\left(\boldsymbol{\Omega}, \boldsymbol{\Omega}^{\prime}, \boldsymbol{B}\right)$, where the explicit form of the Hanle phase matrix, $\mathbf{P}_{\mathrm{H}}$, is given in Stenflo (1994).

\section{Numerical Method}

For the studies presented here we consider CRD, AA-PRD, and AD-PRD. For a static atmosphere, the polarized accelerated lambda iteration techniques to solve the Hanle line transfer equation were developed in Nagendra et al. (1998) for CRD and in Sampoorna et al. (2008) for AA-PRD (given by the so-called Approximation-Ia). The scattering expansion method developed in Nagendra \& Sampoorna (2011) solves the polarized Hanle line transfer equation with AD-PRD (given by the Approximation-I of Bommier 1997). These numerical methods are generalized to include the effects of a vertical velocity field in the observer's frame.

The numerical methods developed in the above-mentioned papers solve the polarized line transfer equation in an irreducible basis (see e.g., Frisch 2007, 2009) in which the line source vector becomes independent of $\boldsymbol{\Omega}$ (in the case of CRD and AA-PRD) and becomes independent of the azimuth, $\chi$, (in the case of AD-PRD). This is true for a 
static atmosphere. In the presence of velocity fields, the CRD line source vector in the irreducible basis continues to be independent of $\Omega$, but the irreducible line source vectors corresponding to both AA- and AD-PRD are only independent of $\chi$. This is because the redistribution functions now depend on $\mu$ and $\mu^{\prime}$, even in the AA-PRD case, since we need to replace $x$ and $x^{\prime}$ with the corresponding Doppler shifted frequencies, namely, $x-\mu V$ and $x^{\prime}-\mu^{\prime} V$. Unlike in CRD, the incoming and outgoing frequencies in PRD are coupled. Thus, the presence of velocity fields brings in an additional coupling between the incoming and outgoing angles not only in the AD-PRD case but also in the AAPRD case. Thus, unlike in the static case, the irreducible line source vector for AA-PRD depends on $\mu$.

The specifications with regard to frequency, depth, and angle griding in the observer's frame are exactly the same as those discussed in Mihalas (1978, see also Carlin et al. 2012). These specifications are over and above those concerning the use of AA- or ADPRD in static media (see Nagendra \& Sampoorna 2011). When depth-dependent velocity fields are used, the redistribution matrix $\mathbf{R}$ becomes depth-dependent, despite assuming an isothermal model atmosphere. Furthermore, to accurately take into account the effects of velocity gradients in the computation of the optical depth increments in the formal solution, it is necessary to use a significantly large number of depth points per decade (say, 20 points per decade). Thus, the requirement of large frequency bandwidths and the fine sampling of the angle and the depth grids, results in a severe demand on both the computing and memory resources. For example, with 81 depth points, 37 frequency points, and 5 Gauss-Legendre angle points, one requires nearly 40 hours of computing time for both AA- and AD-PRD, and a memory of $45 \mathrm{~GB}$ for the former and $95 \mathrm{~GB}$ for the latter. The requirement of significantly more memory for the AD-PRD case comes from the fact that an azimuthal Fourier expansion of the redistribution functions (see Frisch 2009) results in complex irreducible Fourier components of order $k$, which takes values $0, \pm 1, \pm 2, \pm 3, \pm 4$. Thus all the results presented here correspond to - total optical thickness $T=100$ with a lower boundary condition of $I(\tau=T, x, \boldsymbol{\Omega})=1$ and an upper boundary condition of $I(\tau=0, x, \boldsymbol{\Omega})=0, r=10^{-8}, \epsilon=10^{-6}, a=10^{-3}$, and $B_{\nu_{0}}=1$. We neglect the effect of both the line broadening and the depolarizing elastic collisions.

\section{Results and Discussions}

Here we present the emergent Stokes profiles computed for different empirical velocity laws (see Section 4.1). We consider both the non-magnetic (see Section 4.2) and magnetic cases (see Section 4.3). We compare the profiles computed with CRD, AA-PRD, and the AD-PRD assumptions. The static case solution (solid line) is always shown for reference.

\subsection{Empirical Velocity Laws}

For this study we consider moving atmospheres with three types of empirical velocity laws. The first one is an isothermal atmosphere moving with constant velocity towards the observer (i.e., $V(\tau)=$ constant). The second one is an atmosphere with a velocity that varies linearly with optical depth, $V(\tau)=V_{g} \tau$, where $V_{g}$ represents the velocity gradient. Again, a positive velocity gradient is assumed so that the atmosphere is moving towards the observer, however, in this case, different layers are moving with different vertical velocities. The third case is an atmosphere with a velocity law given by (see Mihalas 1978):

$$
V(\tau)=V_{0} /\left[1+\left(\tau / \tau_{0}\right)\right]
$$


(a) CRD
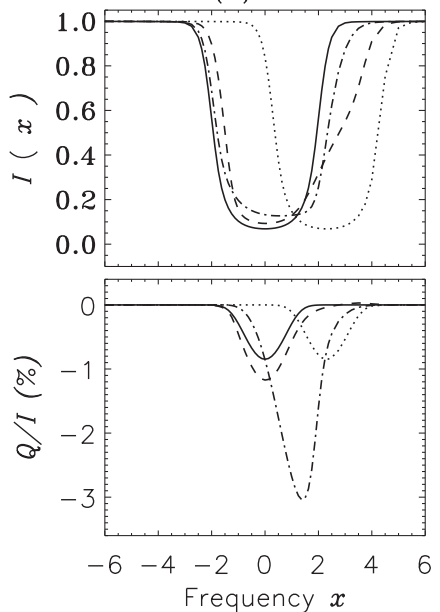

(b) Angle-averaged PRD
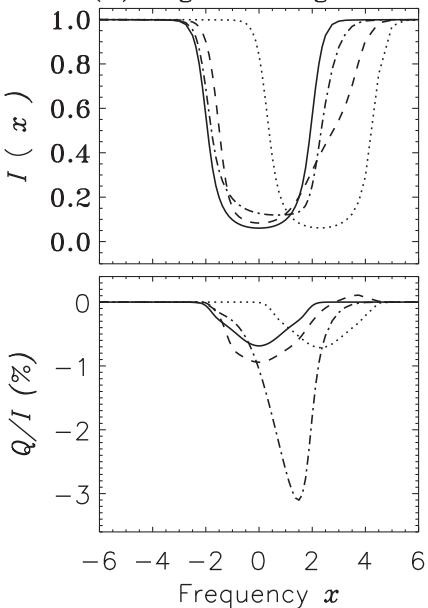

(c) Angle-dependent PRD
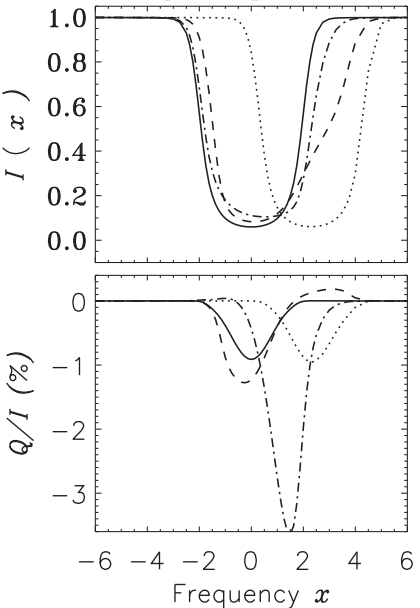

Figure 1. Emergent $(I, Q / I)$ profiles at $\mu=0.769$ from an isothermal atmosphere with model parameters: $T=100, \epsilon=10^{-6}, a=10^{-3}, r=10^{-8}, B_{\nu_{0}}=1$. Panels (a), (b), and (c) show, respectively, the solutions computed with CRD, AA-PRD, and AD-PRD. The solid line corresponds to the static case, the dotted line to $V(\tau)=3$, the dashed line to a linear velocity law with $V_{g}=3 \times 10^{-2}$, and the dot-dashed line to $V(\tau)=V_{0} /\left[1+\left(\tau / \tau_{0}\right)\right]$, with $V_{0}=3$ and $\tau_{0}=1$.

where $V_{0}$ is the limiting value of velocity at small optical depths (i.e., $V_{0}=V(\tau \rightarrow 0)$ ) and $\tau_{0}$ gives the location in optical depth of the largest velocity gradient. Again $V(\tau)$ is taken to be positive, representing an outward motion in the atmosphere.

\subsection{The Case of Non-Magnetic Resonance Scattering}

Here we present the emergent Stokes profiles formed in an isothermal atmosphere in the absence of magnetic fields and with the different velocity laws discussed in Section 4.1. Figure 1 shows the emergent $(I, Q / I)$ profiles for $\mu=0.769$. As expected, we see a Doppler shift of the profiles. A constantly moving atmosphere only produces a bulk Doppler shift, whilst an atmosphere with a velocity gradient, not only produces a Doppler shift, but also enhances the linear polarization compared to the cases of the static and the constantly moving atmospheres. As explained in detail in Carlin et al. (2012) the enhancement in $Q / I$ arises due to an enhancement in anisotropy caused by the Doppler brightening effect. This enhancement in $Q / I$ is progressively larger for AA- and AD-PRD.

Figure 2 shows a comparison between the emergent $Q / I$ profiles computed with ADPRD (solid lines), AA-PRD (dashed lines), and CRD (dotted lines) for $\mu=0.769$. For the chosen model parameters, the intensity profile is not very sensitive to the type of redistribution mechanism used in its computation (see the Stokes $I$ panels in Figure 1), and hence it is not shown. In general the profiles computed with CRD are narrower than those computed with AA- and AD-PRD, except in the case of a moving atmosphere with the velocity law given by Eq. (4.1). The differences between $Q / I$ profiles computed with CRD, AA-PRD, and AD-PRD are of similar nature for the static and the constantly moving atmospheres (compare panels (a) and (b) in Figure 2). Significant differences are seen for a moving atmosphere with a linear velocity law (see Figure 2(c)). In this case the CRD profiles do not exhibit a peak in the blue wing, while the PRD profiles do. Thus, unlike in the cases of the static and the constantly moving atmospheres, AD-PRD effects become important in the case of a moving atmosphere with vertical velocity gradients. 
(a) Static Atmosphere $V(\tau)=0$

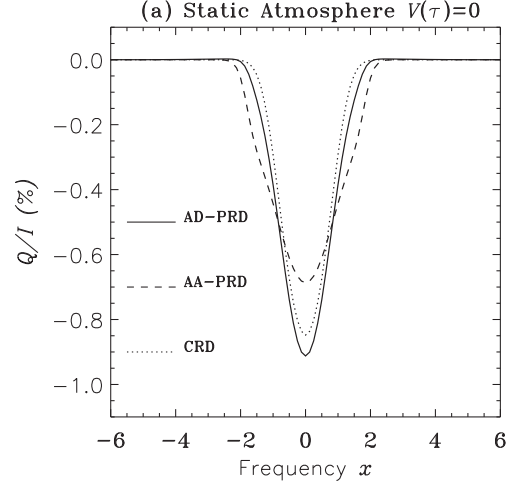

(b) Moving Atmosphere $V(\tau)=3$
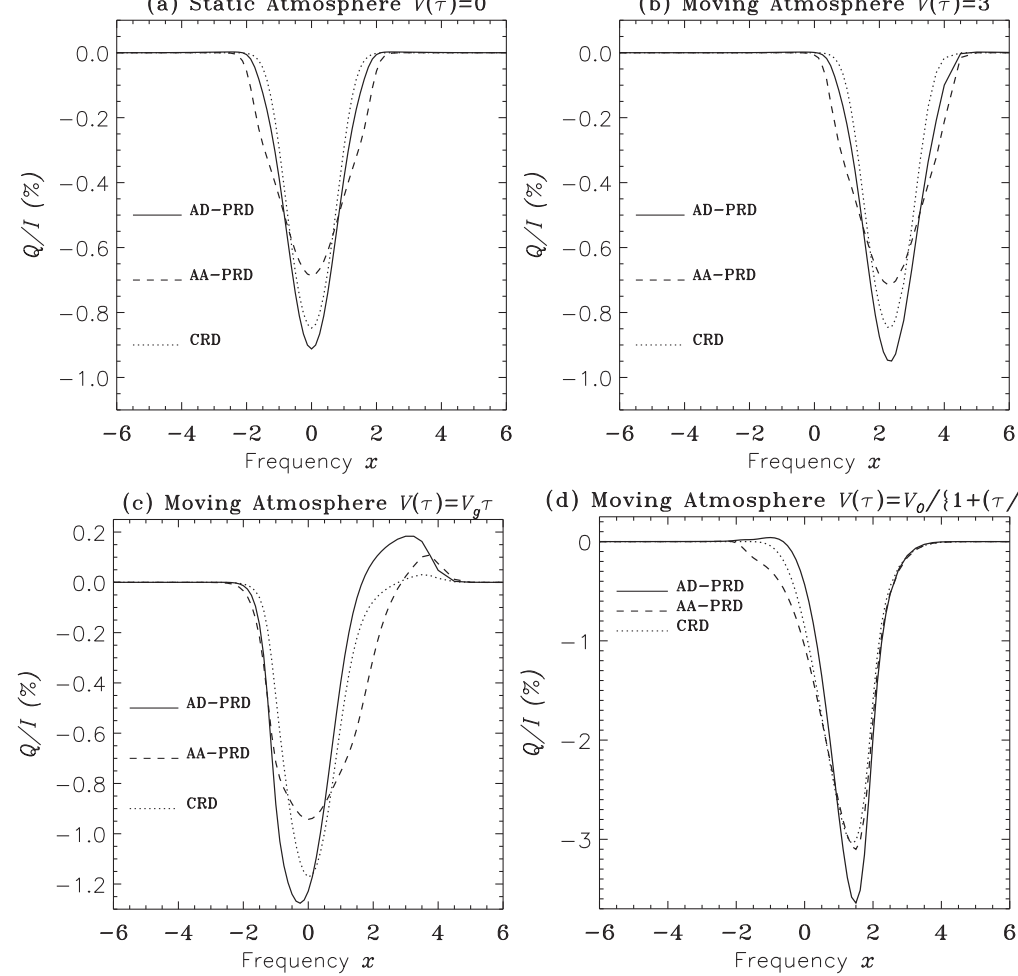

(d) Moving Atmosphere $V(\tau)=V_{0} /\left\{1+\left(\tau / \tau_{0}\right)\right\}$

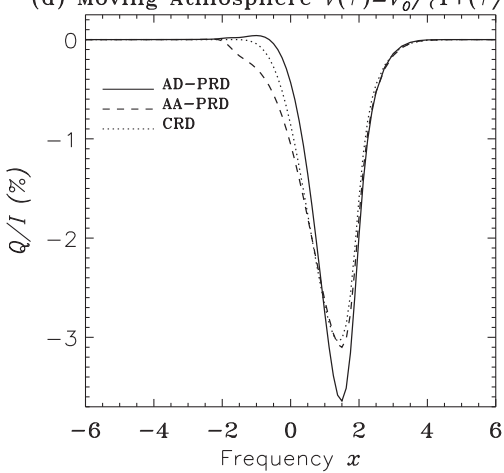

Figure 2. Comparison of emergent $Q / I$ profiles at $\mu=0.769$ computed with AD-PRD, AA-PRD, and CRD. Panels (a)-(d) correspond, respectively, to a static atmosphere, and the three velocity laws discussed in Section 4.1. The model parameters are the same as in Figure 1.

Figure 3 shows the Stokes profiles emerging from a moving atmosphere with a linear velocity law (left) and a velocity law given by Eq. (4.1) (right), again for $\mu=0.769$. Angle-dependent PRD is used in the computations. The figure shows the effects of varying the velocity gradient $V_{g}$ ("a" panels) and the terminal velocity $V_{0}$ ("b" panels). For the case of the linear velocity law, the amplitude of $Q / I$ increases with increasing vertical velocity gradients, and this is accompanied by a Doppler shift in both $I$ and $Q / I$. When assuming the velocity law given by Eq. (4.1), $Q / I$ initially grows with increasing $V_{0}$. Then, it reaches a maximum at $V_{0}=3$ and starts decreasing for larger values of $V_{0}$. This behavior is similar to that discussed in Carlin et al. (2012), who show that the decrease in $Q / I$ for $V_{0}>3$ is due to the larger negative contribution of the horizontal rays to the anisotropy.

\subsection{The Case of Magnetic Hanle Scattering}

Here we present a similar analysis to that presented in Section 4.2 but for a scenario with a weak magnetic field. The magnetic field strength is parametrized through the Hanle $\Gamma_{B}$ parameter, which is defined as $\Gamma_{B}=g_{J_{u}} \omega_{\mathrm{L}} / \Gamma_{\mathrm{R}}$, where $g_{J_{u}}$ denotes the Landé factor of the upper level with total angular momentum $J_{u}, \omega_{\mathrm{L}}$ is the Larmor frequency, and $\Gamma_{\mathrm{R}}$ is the radiative width of the upper level. For all the figures presented in this paper we consider a normal Zeeman triplet with $J_{u}=1$ and $J_{l}=0$. The field inclination and azimuth with respect to the atmospheric normal are denoted by $\theta_{B}$ and $\chi_{B}$ respectively. Figures 4 and 5 are the magnetic analogues of Figures 1 and 2. For all the figures shown in this section, $\Gamma_{B}=1, \theta_{B}=30^{\circ}$, and $\chi_{B}=0^{\circ}$. 

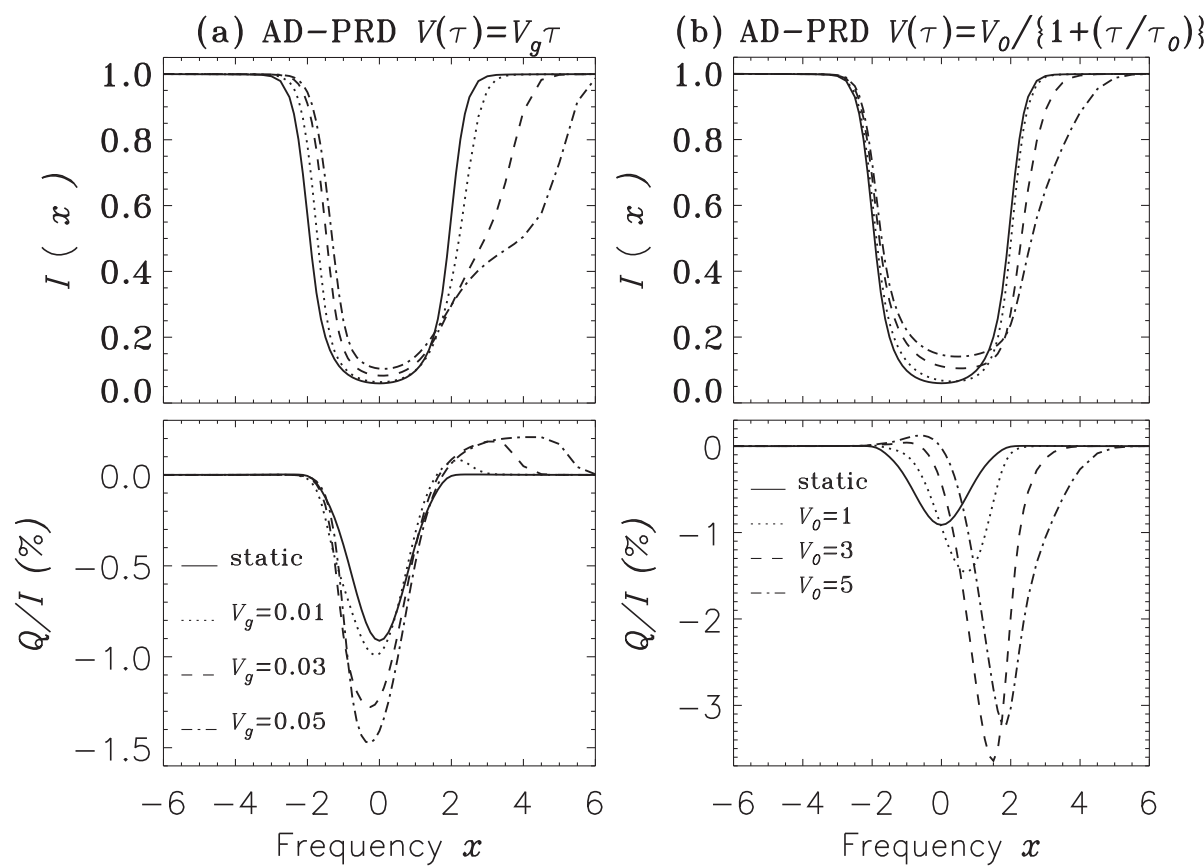

Figure 3. Effects of a linear velocity law (panel a) and the velocity law given by Eq. (4.1) (panel $\mathrm{b}$ ) on the Stokes $I$ and $Q / I$ profiles emerging from a moving atmosphere at $\mu=0.769$. Angle-dependent PRD is used. The remainder of the model parameters are the same as in Figure 1.
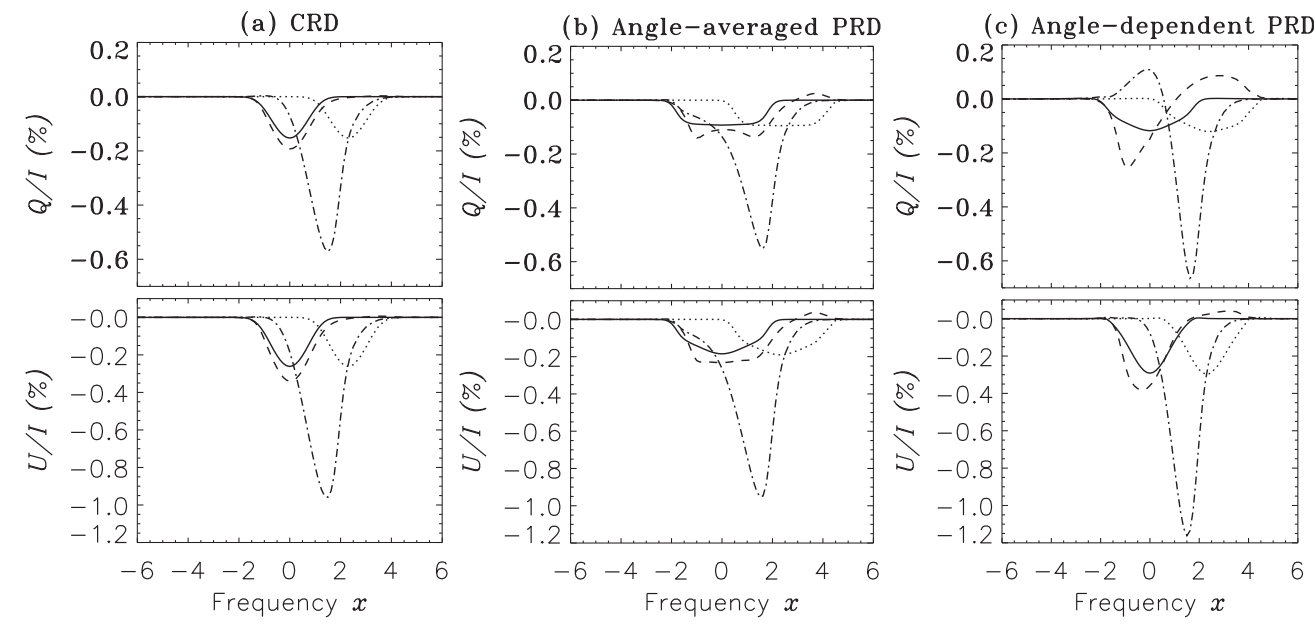

Figure 4. Emergent $(Q / I, U / I)$ profiles at $\mu=0.769$ computed with a weak magnetic field parametrized by $\left(\Gamma_{B}, \theta_{B}, \chi_{B}\right)=\left(1,30^{\circ}, 0^{\circ}\right)$. The remaining model parameters and the line types used in this figure are the same as in Figure 1.

The Stokes $I$ profiles are marginally affected by a weak field, and hence they are not shown here. If we now compare the non-magnetic $Q / I$ profiles shown in Figure 1 with the corresponding magnetic $Q / I$ profiles shown in Figure 4, we notice depolarization in the line core due to the Hanle effect for all cases. For the static and the constantly moving atmospheres the shapes of the $Q / I$ profiles for both the magnetic and the non-magnetic cases are very similar, except for a Hanle depolarization in the magnetic case. For CRD 

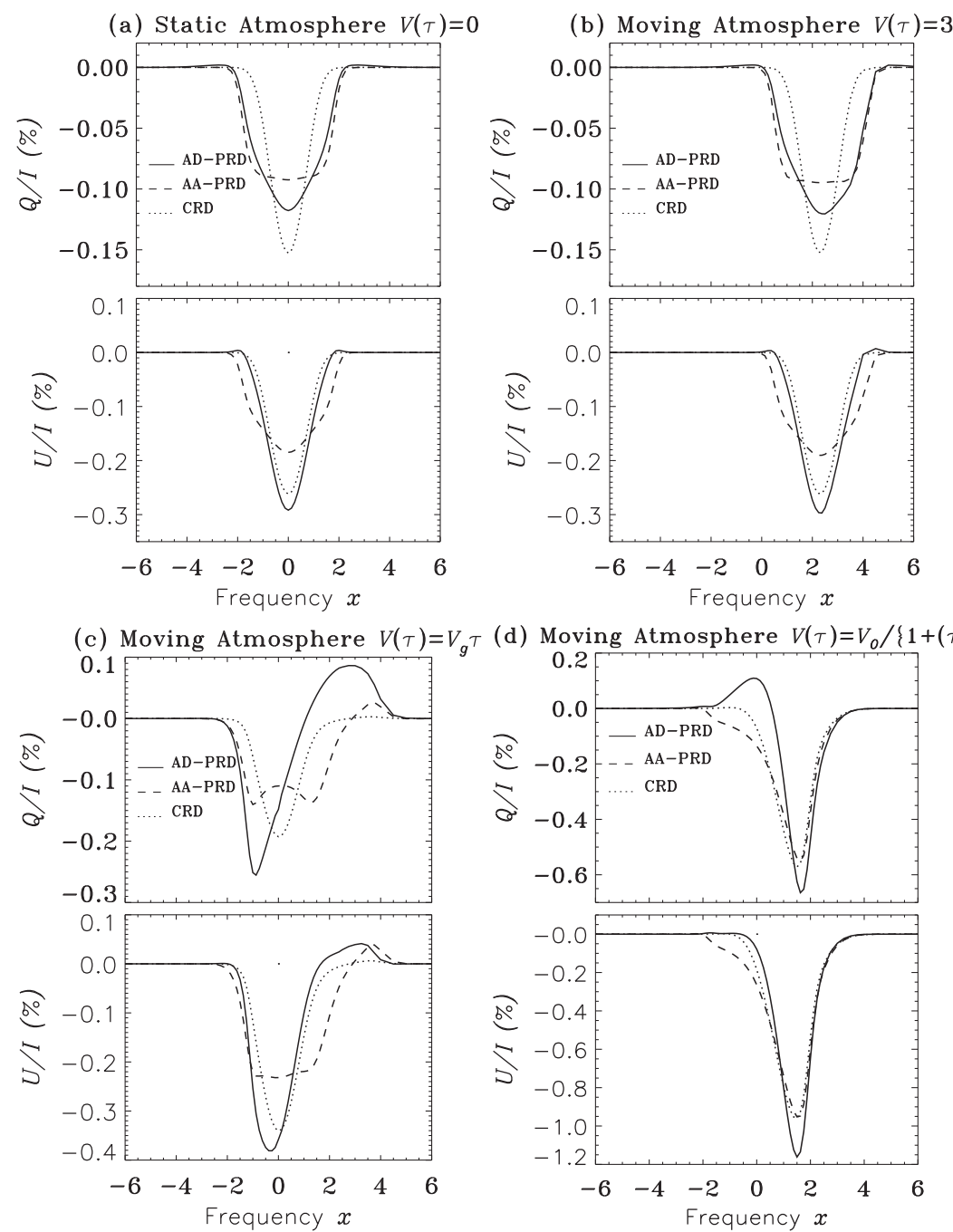

(d) Moving Atmosphere $V(\tau)=V_{0} /\left\{1+\left(\tau / \tau_{0}\right)\right\}$

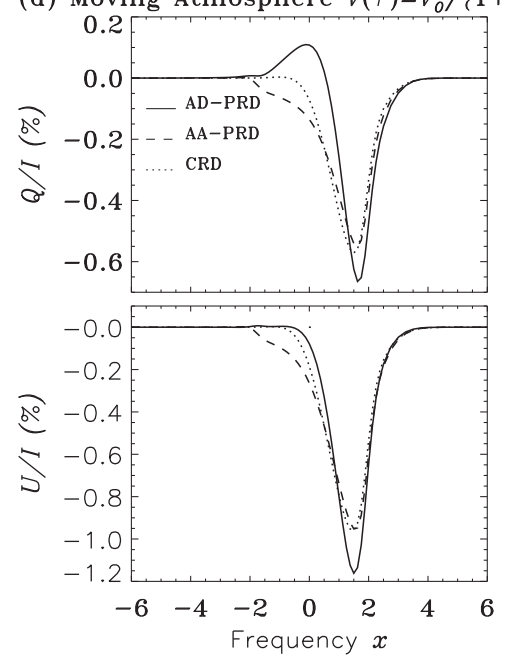

Figure 5. Comparison of the emergent $Q / I$ and $U / I$ profiles at $\mu=0.769$, computed with AD-PRD, AA-PRD, and CRD. Panels (a)-(d) correspond, respectively, to the static atmosphere and the moving atmospheres with the three velocity laws discussed in Section 4.1. The other model parameters are the same as in Figure 4.

and AA-PRD this continues to be valid, even for moving atmospheres with velocity gradients. However, for the AD-PRD case we notice considerable differences in the shapes of the non-magnetic and the magnetic $Q / I$ profiles emerging from an atmosphere with a vertical velocity gradient (compare the dashed and the dot-dashed lines in the $Q / I$ panels of Figures 1(c) and 4(c)). The Stokes $U / I$ profiles are generated due to Hanle rotation. Like the $Q / I$ profiles, they are very sensitive to the vertical velocity gradients.

Figure 5 shows a comparison of the Stokes $Q / I$ and $U / I$ profiles, emerging from a static atmosphere and a moving atmosphere with the three velocity laws, for different redistribution mechanisms. The differences between the magnetic $Q / I$ profiles computed with CRD, AA- and AD-PRD are similar to those observed for the non-magnetic scenario, especially in the cases of the static and the constantly moving atmospheres. For atmospheres with velocity gradients, larger differences can be seen due to the difference in 
the non-magnetic and magnetic $Q / I$ profile shapes computed with AD-PRD (see above). A similar behavior is also exhibited by the $U / I$ profiles. Clearly, the AD-PRD effects are important for both non-magnetic and magnetic cases in the presence of vertical velocity gradients.

\section{Conclusions}

In this paper we have studied the effect of vertical velocity fields on the Stokes profiles emerging from a constant property planar isothermal atmosphere with and without magnetic fields. We have considered the approximation of CRD and both the angleaveraged and angle-dependent PRD. We reinforce the conclusion of Carlin et al. (2012), that, when compared to the static or constantly moving atmospheres, the presence of vertical velocity gradients in the medium enhances the linear polarization and produces Doppler shifted line profiles that are asymmetric about the line center. Our numerical studies show that angle-dependent PRD effects for a optically thin line are important in a moving atmosphere with velocity gradients, both for the non-magnetic and the magnetic cases.

\section{Acknowledgements}

Computations were performed on the HYDRA cluster (dual Xeon X5675 with 6 cores per processor and $3.06 \mathrm{GHz}$ clock speed) and the FORNAX (Dual opteron 6220 with 8 cores and 3.0 GHz clock speed) computing facility at the Indian Institute of Astrophysics.

\section{References}

Bommier, V. 1997, A\&A 328, 726

Carlin, E. S., Asensio Ramos, A., \& Trujillo Bueno, J. 2013, ApJ 764, 40

Carlin, E. S., Manso Sainz, R., Asensio Ramos, A., \& Trujillo Bueno, J. 2012, ApJ 751, 5

Frisch, H. 2007, A\&A 476, 665

Frisch, H. 2009, in: S. V. Berdyugina, K. N. Nagendra \& R. Ramelli (eds.), Solar Polarization 5: In Honor of Jan Stenflo, ASP Conf. Series 405 (San Francisco: ASP), p. 87

Mihalas, D. 1978, Stellar Atmospheres, Freeman, San Francisco

Nagendra, K. N. 1996, Solar Phys. 164, 67

Nagendra, K. N., Frisch, H., \& Faurobert-Scholl, M. 1998, A $\& A$ 332, 610

Nagendra, K. N. \& Sampoorna, M. 2011, A\&4A 535, A88

Sampoorna, M. 2014, in: K. N. Nagendra, J. O. Stenflo, Z. Q. Qu \& M. Sampoorna (eds.), Solar Polarization 7, ASP Conf. Series 489 (San Francisco: ASP), p. 197

Sampoorna, M., Nagendra, K. N., \& Frisch, H. 2008, JQSRT 109, 2349

Sengupta, S. 1993, MNRAS 265, 513

Stenflo, J. O. 1994, Solar magnetic fields - Polarized radiation diagnostics, Kluwer, Dordrecht 